OPEN ACCESS

Edited by:

Panteleimon Giannakopoulos,

Université de Genève, Switzerland

Reviewed by:

Ayodeji A. Asuni,

New York University, United States Ignacio Torres-Aleman,

Consejo Superior de Investigaciones

Cientificas (CSIC), Spain

*Correspondence:

Linda J. Van Eldik

linda.vaneldik@uky.edu

Received: 27 April 2018

Accepted: 17 August 2018

Published: 11 September 2018

Citation:

Braun DJ and Van Eldik LJ (2018)

In vivo Brainstem Imaging

in Alzheimer's Disease: Potential

for Biomarker Development.

Front. Aging Neurosci. 10:266.

doi: 10.3389/fnagi.2018.00266

\section{In vivo Brainstem Imaging in Alzheimer's Disease: Potential for Biomarker Development}

\author{
David J. Braun ${ }^{1}$ and Linda J. Van Eldik ${ }^{1,2,3 *}$ \\ ' Sanders-Brown Center on Aging, University of Kentucky, Lexington, $K Y$, United States, ${ }^{2}$ Spinal Cord and Brain Injury \\ Research Center, University of Kentucky, Lexington, KY, United States, ${ }^{3}$ Department of Neuroscience, University \\ of Kentucky, Lexington, KY, United States
}

The dearth of effective treatments for Alzheimer's disease (AD) is one of the largest public health issues worldwide, costing hundreds of billions of dollars per year. From a therapeutic standpoint, research efforts to date have met with strikingly little clinical success. One major issue is that trials begin after substantial pathological change has occurred, and it is increasingly clear that the most effective treatment regimens will need to be administered earlier in the disease process. In order to identify individuals within the long preclinical phase of $A D$ who are likely to progress to dementia, improvements are required in biomarker development. One potential area of research that might prove fruitful in this regard is the in vivo detection of brainstem pathology. The brainstem is known to undergo pathological changes very early and progressively in AD. With an updated and harmonized $A D$ research framework, and emerging advances in neuroimaging technology, the potential to leverage knowledge of brainstem pathology into biomarkers for $\mathrm{AD}$ will be discussed.

Keywords: Alzheimer's disease, brainstem, neuroimaging, imaging, in vivo, locus coeruleus, raphe nucleus, biomarker

\section{INTRODUCTION}

Dementia is a global public health crisis, affecting around 47 million people worldwide and projected to nearly triple by 2050 as the global population increases in average age (World Health Organization, 2017). Alzheimer's disease (AD) is the most common cause of aging-related dementia, accounting for $60-80 \%$ of cases (Alzheimer's Association, 2017). Even a relatively small reduction or delay in $\mathrm{AD}$ incidence therefore has the potential to dramatically reduce dementia burden (Barnes and Yaffe, 2011). Unfortunately, current AD treatments are only moderately and transiently effective, and many promising treatments have fallen short in late-stage clinical trials (Mehta et al., 2017). One major obstacle is the need for refined early-stage biomarkers that can reliably predict who within the AD spectrum will develop dementia. The brainstem has long been recognized as a site with early and significant susceptibility to AD-type neuropathological change (Mann, 1983), but it has been difficult to access this region in vivo. Advances in non-invasive neuroimaging techniques are beginning to allow antemortem assessment of this crucial region, thereby opening the possibility of using early brainstem changes as $\mathrm{AD}$ biomarkers. This might extend the window in which preclinical $\mathrm{AD}$ is detectable or, in conjunction with other biomarkers, better define those patients most likely to experience cognitive dysfunction in the future. This 
concept will be discussed in light of the current $\mathrm{AD}$ research framework and recent neuroimaging advances.

\section{ALZHEIMER'S DISEASE PATHOLOGY AND DIAGNOSTIC CRITERIA}

The essential neuropathologic features of $\mathrm{AD}$ consist of extracellular deposits of amyloid beta peptide $(A \beta)$, and intracellular accumulations of modified tau protein called neurofibrillary tangles (NFTs). These pathologies are accompanied by neuronal injury and neurodegeneration, leading to progressive cognitive impairment and culminating in dementia. An updated clinical staging of $\mathrm{AD}$ was defined in 2011 by a joint workgroup of the National Institute on Aging and the Alzheimer's Association (NIA-AA) (Albert et al., 2011; McKhann et al., 2011; Sperling et al., 2011; Montine et al., 2012). Associated with this, the NIA-AA published neuropathologic guidelines to score $\mathrm{AD}$-associated neuropathology as well as assess commonly appearing comorbid pathologies (Montine et al., 2012). Importantly, the 2011 guidelines also formalized the use of imaging and cerebrospinal fluid (CSF) biomarkers to define "preclinical AD," wherein an individual has abnormal AD-type pathological changes and no or only subtle cognitive/behavioral symptoms. Validated in vivo markers for $\mathrm{AD}$-type neuropathologic change include CSF levels of $A \beta_{42}$ and phosphorylated tau, increased positron emission tomography (PET) ligand binding for amyloid or tau within the cortex, glucose hypometabolism as measured by fluorodeoxyglucose PET (FDG-PET), and brain atrophy as measured by structural magnetic resonance imaging (MRI) (Tan et al., 2014). This biomarker-defined preclinical AD framework is particularly useful as the preclinical phase can extend more than a decade before the onset of clinically defined symptoms (Quiroz et al., 2018), and therefore represents a promising window for therapeutic intervention.

The disparity between reference to $\mathrm{AD}$ as a clinical syndrome versus a neuropathologic phenomenon has led to some confusion, however. For example, around $30 \%$ of patients clinically diagnosed with $\mathrm{AD}$ do not have significant $\mathrm{AD}$ neuropathology at autopsy and a similar proportion of cognitively unimpaired patients do (Davis et al., 1999; Neuropathology Group, 2001). In an attempt to harmonize definitions and clarify research efforts, the NIA-AA recently released an updated 2018 framework, building upon the 2011 biomarker-based approach in defining preclinical $\mathrm{AD}$ (Jack et al., 2018; Khachaturian et al., 2018; Knopman et al., 2018; Silverberg et al., 2018). This new system categorizes all patients by the presence or absence of biomarker-based pathology in three categories to generate an "ATN" profile: A for amyloid, $\mathrm{T}$ for tau, and $\mathrm{N}$ for neurodegeneration. Additionally, there is a cognitive staging scheme of cognitively unimpaired, mild cognitive impairment (MCI), or dementia overlaid on the ATN biomarker profiles (Table 1). An individual must have biomarker group A pathology (reduced CSF $A \beta_{42}$, increased amyloid PET, etc.) to be placed on the $\mathrm{AD}$ spectrum, irrespective of cognitive or other pathological changes. The framework is flexible in that it can incorporate new ATN biomarkers as they become validated, or entirely new biomarker categories. As will be discussed below, neuropathological data indicate that neuroimaging-accessible changes within the brainstem may be useful additions to the $\mathrm{T}$ or $\mathrm{N}$ biomarker groupings.

\section{BRAINSTEM CHANGES IN AD}

Although considerable focus in the AD field has been placed on pathological changes in cortical, hippocampal, and basal forebrain regions, brainstem pathology has also been described since at least the 1930s (Hannah, 1936). The brainstem is a small and complex region, serving as a major relay center and signal integrator for the central nervous system (CNS). Rostro-caudally it is comprised of the midbrain, pons, and medulla, together containing the majority of neurons belonging to several widely projecting monoaminergic modulatory systems. These monoamine transmitters include serotonin (5-HT) and the catecholamines dopamine and noradrenaline (NA), the dysfunction of which have been well-described in AD (Trillo et al., 2013; Šimić et al., 2017). Early and severe ADassociated changes occur in the major brainstem serotonergic and noradrenergic nuclei, the dorsal raphe nucleus (DRN) and locus coeruleus (LC), respectively, a brief summary of which is provided below. For more in depth reading, several comprehensive reviews have been published (Szabadi, 2013; Trillo et al., 2013; Giorgi et al., 2017; Šimić et al., 2017).

\section{The Dorsal Raphe Nucleus}

The DRN is one of a cluster of serotonergic nuclei, located near the midline in the dorsal midbrain and pons. It contains the majority of brainstem serotonergic neurons and is comprised of about 235,000 neurons in the adult human brain, roughly $70 \%$ of which produce 5-HT. The DRN sends ascending projections to regions such as the cortex, hippocampus, and striatum, as well as descending projections to the lower brainstem and spinal cord (Šimić et al., 2017). The serotonergic neurons of the DRN undergo substantial cell loss in AD (Lyness et al., 2003), and tangle pathology occurs early in the disease process (Rüb et al., 2000; Grinberg et al., 2009; Braak et al., 2011; Ehrenberg et al., 2017). Dysfunction in the serotonergic system has been strongly implicated in the behavioral and psychological symptoms of dementia (BPSD), such as agitation, aggression, hallucinations, and depression (Lanctôt et al., 2001). In particular, depressive symptoms later in life appear to be a feature of preclinical neurodegenerative changes, even before the onset of cognitive decline (Donovan et al., 2015; SinghManoux et al., 2017). Similarly, the DRN (along with the LC) is heavily involved in regulation of the sleep/wake cycle and cognitively normal individuals meeting criteria for preclinical AD have measurably worse sleep quality versus controls (Ju et al., 2013).

\section{The Locus Coeruleus}

The LC is a long and narrow nucleus in the brainstem pons, averaging about $14.5 \mathrm{~mm}$ long and up to about $2.5 \mathrm{~mm}$ 
TABLE 1 | Overview of NIA-AA ATN biomarker scores and cognitive staging.

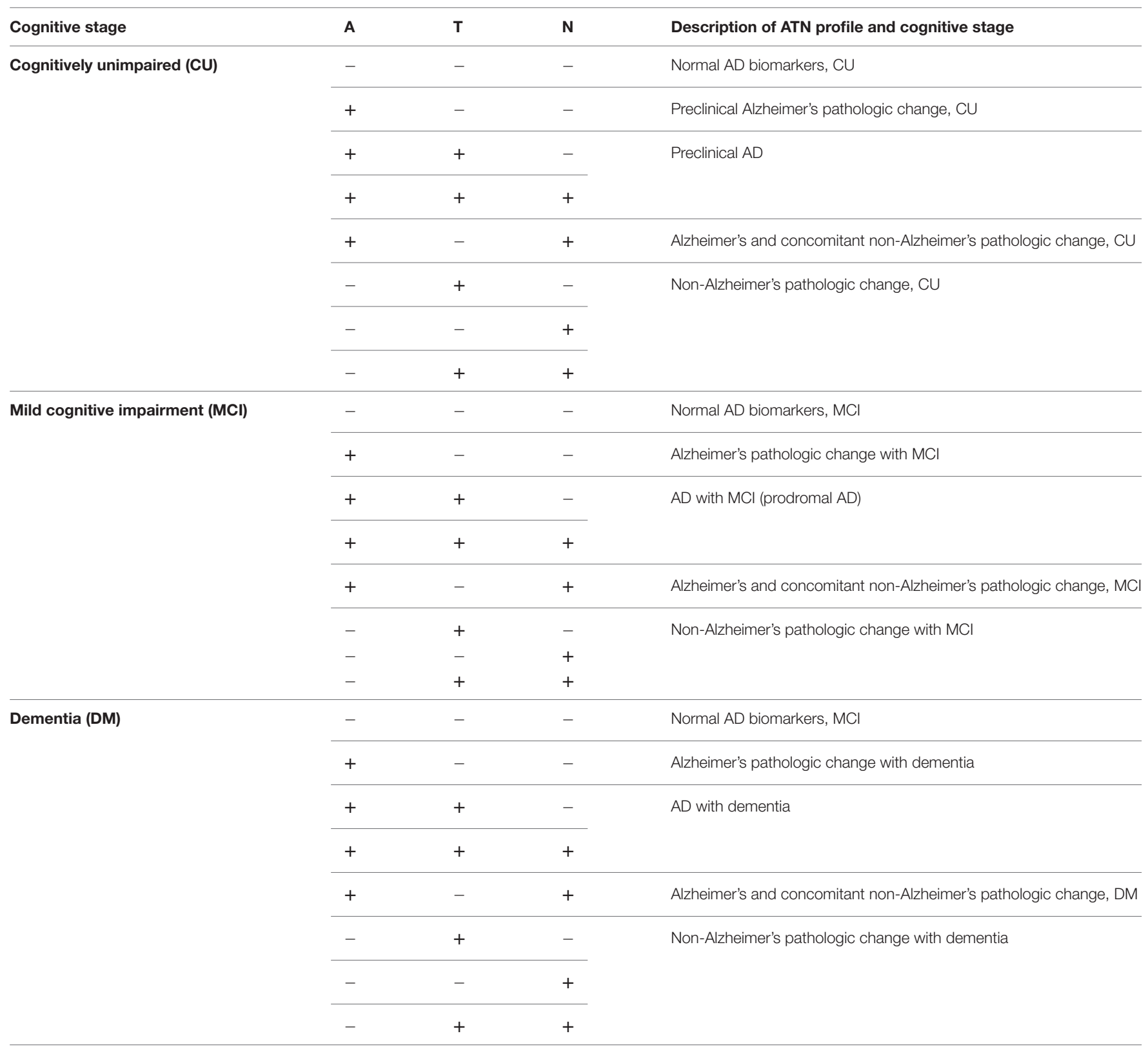

Only those with A biomarkers $(A+)$ are considered to be on the AD pathological spectrum. Biomarkers for $A$ (amyloid) include CSF A $\beta_{42}$ and amyloid PET. Biomarkers for $T$ (tau) include CSF p-tau and tau PET. Biomarkers for N (neurodegeneration) include CSF total tau, structural changes detectable by MRI (i.e., atrophy), and FDG-PET. Neuroimaging-detectable brainstem changes might inform the T or N categories, or potentially comprise a separate category in future iterations of the NIA-AA research framework.

thick (Fernandes et al., 2012). It extends caudally along the cerebral aqueduct from the level of the inferior colliculus, ending ventrolateral to the floor of the fourth ventricle. It consists of only about 20 to 30,000 neurons per side in the healthy adult (German et al., 1988; Baker et al., 1989). Despite its small size, the LC provides the majority of central noradrenergic innervation via extensive projections throughout the CNS and it plays important roles across behavioral, cognitive, and physiological domains. It has also been implicated in early BPSD changes (Matthews et al., 2002) and tangle pathology in the LC occurs early and progressively throughout the disease process, even prior to tangle pathology within the DRN (Ehrenberg et al., 2017). Interestingly, the first detectable tau pathology within the brain is in the LC, appearing even in cognitively normal young individuals (Braak et al., 2011), and it has been speculated that LC damage and noradrenergic dysfunction might potentiate pathology in target regions such as cortex and hippocampus (Heneka et al., 2010; Braun et al., 2014; Feinstein et al., 2016). The LC undergoes severe topographic degeneration in $\mathrm{AD}$, such that areas containing 
neurons reciprocally connected to hippocampal and cortical forebrain regions are selectively lost (Marcyniuk et al., 1986; Lyness et al., 2003; Zarow et al., 2003; Theofilas et al., 2017), and LC degeneration correlates with cognitive dysfunction (Grudzien et al., 2007). It is important to note here that the distinct but common condition known as primary agerelated tauopathy (PART) also manifests with early LC tau pathology (Nelson et al., 2016). AD-related LC tau pathology by definition refers only to that co-occurring with amyloid biomarker changes (e.g., reduced CSF $\mathrm{A} \beta_{42}$, increased cortical amyloid PET).

Although the DRN and LC are quite small, the pathology within them occurs early, progressively, and severely, increasing the likelihood of neuroimaging-based detection in the preclinical phase of disease. How this might practically be achieved is the topic of the following section.

\section{NEUROIMAGING THE BRAINSTEM}

Of the non-invasive imaging modalities available, MRI and PET are those most commonly used in AD research (Table 2). MRI is primarily used to rule out other causes of dementia (e.g., stroke) and assess brain structural alterations informative of neurodegenerative change (Kehoe et al., 2014). It can also measure functional parameters relevant to AD pathology such as tissue perfusion (Wolk and Detre, 2012) or blood brain barrier (BBB) integrity (Raja et al., 2017); however, these methods have yet to be validated for biomarker use. $\mathrm{PET}$ in the $\mathrm{AD}$ context is employed primarily for assessment of levels of $A \beta$ or tau, and to measure brain glucose hypometabolism with FDG-PET (Bao et al., 2017; Rice and Bisdas, 2017; Villemagne et al., 2018). These measurements are taken in relevant cortical and hippocampal regions, due in part to logistical considerations that have limited the use of these modalities for brainstem evaluation. The brainstem is a small structure with substantial anatomical complexity, requiring high resolution to visualize its component structures, and there is little endogenous contrast to enable the easy separation of nuclei. Additionally, significant physiological noise is present from cardiorespiratory systems, making fine-grained measurements difficult even with higher resolution systems (for review see Sclocco et al., 2017). Nonetheless, refined techniques are increasing the sensitivity of brainstem measurement, and a recent MRI study successfully identified reductions in dorsal midbrain volume in $\mathrm{AD}$ patients, corresponding to the location of the raphe nuclei (Lee et al., 2015). The increasing adoption of ultra-high-field (UHF) magnets for MRI (Deistung et al., 2013) and the High-Resolution Research Tomograph (HRRT) for PET (Schain et al., 2013), along with improved analytical methods (Iglesias et al., 2015), are enhancing the accessibility of these small brainstem regions to neuroimaging.

\section{Neuroimaging the DRN}

The imaging of individual brainstem raphe nuclei poses some challenges (Kranz et al., 2012). However, it has primarily been achieved with the use of PET radioligands specific for 5HT receptors or the 5-HT transporter (Kranz et al., 2012; Paterson et al., 2013; Schain et al., 2013; Kumar and Mann, 2014; Zhang et al., 2017). There are only two studies directly assessing the $\mathrm{DRN}$ in vivo in $\mathrm{AD}$ spectrum changes, both using functional MRI (fMRI). In one, reduced default mode network connectivity of the DRN was found in patients with $\mathrm{AD}$, distinctive from changes observed in dementia with Lewy bodies (Zhou et al., 2010). A combined PET and fMRI study found reduced 5-HT transporter in the DRN of patients with MCI versus controls, associated with reduced functional connectivity between the hippocampus and DRN (Barrett et al., 2017). In addition, neuroimaging alterations in DRN are observable in patients with anxiety (Lanzenberger et al., 2007; Lee et al., 2011; Johnston et al., 2015; Pillai et al., 2018) and major depressive disorder (Lee et al., 2011; Johnston et al., 2015; Pillai et al., 2018), indicating that such changes might also be detectable in patients with BPSD due to $\mathrm{AD}$. Interestingly, one group has successfully used a fusion PET/MRI system with both HRRT PET and UHF MRI (7T) for brainstem serotonergic imaging (Cho et al., 2007). With this system, all 5 individual raphe nuclei have been visualized using UHF MRI and FDG-PET or ${ }^{11} \mathrm{C}$-DASB PET (a radioligand for the 5-HT transporter) (Son et al., 2014). This raises the immediate possibility of testing the hypothesis that neurodegenerative biomarker changes may be observable in the DRN in early stages of $\mathrm{AD}$. Whether such a system might also be able to detect other pathology (e.g., tau PET) in the DRN remains to be seen, but such a study would be well worth the effort. A major caveat is that the current cost and rarity of such systems limit their use; however, they are likely to become more common with time (Nensa et al., 2014), especially in light of demonstrated utility in dementia research (Dukart et al., 2011). Specific funding to equip Alzheimer's Disease Research Centers with such systems would rapidly expand their use and facilitate research in this domain.

\section{Neuroimaging the LC}

The biosynthesis of catecholamines in humans is associated with the production of neuromelanin, putatively a mechanism to reduce oxidative damage (Zecca et al., 2008). The noradrenergic LC is no exception, with neuromelanin levels increasing with age and then starting to decline around the seventh decade of life (Mann and Yates, 1974). Conveniently, neuromelanin has paramagnetic properties that allow regions with a high neuromelanin content to be directly imaged with T1-weighted or magnetization transfer weighted MRI scans on widely used 3T magnets (Sasaki et al., 2006, 2008). This neuromelaninsensitive MRI (NM-MRI) allows for reliable mapping of the human LC (Keren et al., 2009, 2015; Tona et al., 2017). Although this property is increasingly being exploited, only two studies have applied this approach to $\mathrm{AD}$ patients in vivo. In one study, a non-significant reduction in neuromelanin signal versus controls was found, however, only $6 \mathrm{AD}$ patients were included in the study (Miyoshi et al., 2013). A subsequent larger study of $22 \mathrm{AD}, 38 \mathrm{MCI}$, and 26 controls reported 
TABLE 2 | Validated and potential neuroimaging-based biomarkers for AD-relevant pathology.

\begin{tabular}{|c|c|c|}
\hline & Pathology & Imaging technique/sequence \\
\hline \multirow[t]{4}{*}{ Validated AD neuroimaging biomarkers ${ }^{1}$} & Amyloid (A) & Amyloid PET \\
\hline & Tau $(T)$ & Tau PET \\
\hline & Hypometabolism (N) & FDG-PET \\
\hline & Atrophy $(\mathrm{N})$ & Structural MRI \\
\hline \multirow[t]{8}{*}{ Potential AD neuroimaging biomarkers } & Perfusion & Arterial spin labeling, contrast-enhanced MRI \\
\hline & BBB integrity & Contrast-enhanced MRI \\
\hline & Vascular reactivity & Blood-oxygen-level-dependent MRI \\
\hline & Functional connectivity & Blood-oxygen-level-dependent MRI \\
\hline & Neurotransmitter dysfunction & Neuromelanin-sensitive MRI, MRS, PET \\
\hline & White matter disruption & Diffusion tensor imaging \\
\hline & Neuroinflammation & MRS, translocator protein PET* \\
\hline & Synaptic dysfunction & Synaptic vesicle glycoprotein 2A PET* \\
\hline
\end{tabular}

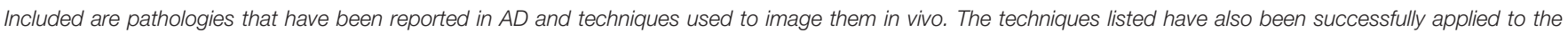
brainstem in the published literature in various contexts, with the exception of the newer PET indicators of neuroinflammation and synaptic dysfunction, marked by*. MRS, magnetic resonance spectroscopy. ${ }^{1}$ Validated AD neuroimaging biomarkers are those described in the recently updated NIA-AA research framework (Jack et al., 2018).

significantly reduced LC neuromelanin signal in both MCI and $\mathrm{AD}$ patients as compared with controls, with no difference between MCI or AD groups (Takahashi et al., 2015). This latter finding indicates that neuronal loss might occur relatively early in these patients, even before clinically detectable cognitive impairment. Interestingly, a study of healthy individuals showed that the LC neuromelanin signal positively correlates with established proxies of neural reserve such as years of education (Clewett et al., 2016), a finding in line with other data that implicate the LC as a physiological substrate of cognitive reserve (Robertson, 2013; Wilson et al., 2013). Further, another recent study found that reduced LC neuromelanin signal was associated with poorer memory even in healthy older adults, particularly for emotionally negative events (Hämmerer et al., 2018). Such findings raise the possibility that LC neuromelanin signal may have prognostic value for older individuals in preclinical stages of $\mathrm{AD}$, well before cognitive impairment.

In addition to NM-MRI, there have been studies examining LC functional connectivity in healthy and clinical populations. These studies have primarily focused on psychiatric symptoms, showing that LC functional connectivity with certain limbic regions is increased in patients with generalized anxiety disorder (Meeten et al., 2016), post-traumatic stress disorder (Steuwe et al., 2015), or those at high risk for schizophrenia (Anticevic et al., 2014). Whether such changes are also present in AD patients with BPSD has yet to be determined. Additionally, PET radioligands have been developed that bind the noradrenaline transporter (NET) (Stehouwer and Goodman, 2009; Adhikarla et al., 2016) and various adrenoceptor subtypes (Lehto et al., 2015; Phan et al., 2017). These have even been combined with NM-MRI, in a study demonstrating a correlation between LC neuromelanin signal and NET binding in certain LC projection areas in Parkinson's disease (Sommerauer et al., 2018). Although various studies have found that NET density is decreased within the LC of the AD brain (Tejani-Butt et al., 1993; Szot et al., 2000; Gulyás et al., 2010), this has yet to be reported in $\mathrm{AD}$ patients in vivo.

\section{SUMMARY}

Thanks to recent advances in neuroimaging technologies, particularly UHF MRI, HRRT PET, and hybrid PET/MRI systems, the capacity to measure $\mathrm{AD}$-associated brainstem pathology in vivo has reached a point where we can begin to assess its utility. Given the neuropathological findings described above, it is reasonable to expect alterations in these regions in at least a subset of patients with preclinical $\mathrm{AD}$ or even preclinical Alzheimer's pathologic change (Table 1). One of the first questions to address is whether neuroimagingdetectable LC or DRN pathology, either alone or in some combination with other markers, can help predict which patients on the $\mathrm{AD}$ spectrum will go on to develop $\mathrm{AD}$ dementia. The LC in particular seems promising in light of the aforementioned evidence indicating its early loss in size (Theofilas et al., 2017) and its potential role as a substrate of cognitive reserve (Wilson et al., 2013; Clewett et al., 2016; Hämmerer et al., 2018). Additionally, the technology to assess its integrity in vivo is already in increasingly widespread use. Indeed, a recent systematic review of LC NM-MRI studies by Liu et al. (2017) identified areas where this technique can be improved, particularly in terms of standardization of LC signal measurements and methods of sub-regional analysis. This latter aspect may be especially useful given the topographic specificity of LC degeneration in AD. Additionally, the advent of new PET radioligands for synaptic dysfunction and neuroinflammatory change (Bao et al., 2017; Table 2) opens new possibilities. Neuroinflammation in particular is increasingly appreciated for its significant role in the early etiology of $\mathrm{AD}$, and various MRS-detectable metabolites have historically been taken as indicators of neuroinflammatory changes (Quarantelli, 2015). More recently, progress has been 
made in PET imaging of translocator protein (TSPO) as a biomarker of activated microglia and astrocytes (Kreisl et al., 2018).

Despite the promise, there is much to be studied before such strategies might be employed in the clinic. The aforementioned studies have been small in both size and number, and they need to be expanded and replicated. A more fundamental question of whether brainstem neuroimaging is likely to provide benefit above and beyond other biomarkers remains and, while it is too early to say, we believe that the potential is worth investigating as technological advancements allow it. Finally, whether or not neuroimaging within the LC or DRN becomes useful clinically, the techniques outlined here afford an opportunity to advance our understanding not only of $\mathrm{AD}$

\section{REFERENCES}

Adhikarla, V., Zeng, F., Votaw, J., Goodman, M., and Nye, J. (2016). Compartmental modeling of [11C]MENET binding to the norepinephrine transporter in the healthy human brain. Nucl. Med. Biol. 43, 318-323. doi: 10.1016/j.nucmedbio.2016.02.008

Albert, M., DeKosky, S., Dickson, D., Dubois, B., Feldman, H., Fox, N., et al. (2011). The diagnosis of mild cognitive impairment due to Alzheimer's disease: recommendations from the National Institute on Aging-Alzheimer's association workgroups on diagnostic guidelines for Alzheimer's disease. Alzheimers Dement. 7, 270-279. doi: 10.1016/j.jalz.2011.03.008

Alzheimer's Association (2017). Alzheimer's disease facts and figures. Alzheimers Dement. 13, 325-373. doi: 10.1016/j.jalz.2017.02.001

Anticevic, A., Tang, Y., Cho, Y., Repovs, G., Cole, M., Savic, A., et al. (2014). Amygdala connectivity differs among chronic, early course, and individuals at risk for developing schizophrenia. Schizophr. Bull. 40, 1105-1116. doi: 10.1093/ schbul/sbt165

Baker, K. G., Törk, I., Hornung, J. P., and Halasz, P. (1989). The human locus coeruleus complex: an immunohistochemical and three dimensional reconstruction study. Exp. Brain Res. 77, 257-270. doi: 10.1007/BF00274983

Bao, W., Jia, H., Finnema, S., Cai, Z., Carson, R., and Huang, Y. (2017). PET Imaging for early detection of Alzheimer's disease. PET Clin. 12, 329-350. doi: 10.1016/j.cpet.2017.03.001

Barnes, D., and Yaffe, K. (2011). The projected effect of risk factor reduction on Alzheimer's disease prevalence. Lancet Neurol. 10, 819-828. doi: 10.1016/ S1474-4422(11)70072-2

Barrett, F., Workman, C., Sair, H., Savonenko, A., Kraut, M., Sodums, D., et al. (2017). Association between serotonin denervation and resting-state functional connectivity in mild cognitive impairment. Hum. Brain Mapp. 38, 3391-3401. doi: 10.1002/hbm.23595

Braak, H., Thal, D. R., Ghebremedhin, E., and Del Tredici, K. (2011). Stages of the pathologic process in Alzheimer disease: age categories from 1 to 100 years. J. Neuropathol. Exp. Neurol. 70, 960-969. doi: 10.1097/NEN.0b013e318232a379

Braun, D., Madrigal, J. L., and Feinstein, D. L. (2014). Noradrenergic regulation of glial activation: molecular mechanisms and therapeutic implications. Curr. Neuropharmacol. 12, 342-352. doi: 10.2174/1570159X1266614082822 0938

Cho, Z., Son, Y., Kim, H., Kim, K., Oh, S., Han, J., et al. (2007). A hybrid PET-MRI: an integrated molecular-genetic imaging system with HRRT-PET and 7.0-T MRI. Int. J. Imaging Syst. Technol. 17, 252-265. doi: 10.1002/ima. 20119

Clewett, D., Lee, T.-H., Greening, S., Ponzio, A., Margalit, E., and Mather, M. (2016). Neuromelanin marks the spot: identifying a locus coeruleus biomarker of cognitive reserve in healthy aging. Neurobiol. Aging 37, 117-126. doi: 10.1016/j.neurobiolaging.2015.09.019

Davis, D. G., Schmitt, F. A., Wekstein, D. R., and Markesbery, W. R. (1999). Alzheimer neuropathologic alterations in aged cognitively normal subjects. J. Neuropathol. Exp. Neurol. 58, 376-388. doi: 10.1097/00005072-19990400000008 pathological progression, but other neurodegenerative diseases as well.

\section{AUTHOR CONTRIBUTIONS}

DB wrote the manuscript. All authors conceived, revised, and approved the final manuscript.

\section{FUNDING}

This work was supported in part by the Weston Brain Institute and National Institutes of Health (F32-AG058456).

Deistung, A., Schäfer, A., Schweser, F., Biedermann, U., Güllmar, D., Trampel, R., et al. (2013). High-resolution MR imaging of the human brainstem In vivo at 7 Tesla. Front. Hum. Neurosci. 7:710. doi: 10.3389/fnhum.2013.00710

Donovan, N., Hsu, D., Dagley, A., Schultz, A., Amariglio, R., Mormino, E., et al. (2015). Depressive symptoms and biomarkers of Alzheimer's Disease in cognitively normal older adults. J. Alzheimers Dis. 46, 63-73. doi: 10.3233/JAD142940

Dukart, J., Mueller, K., Horstmann, A., Barthel, H., Möller, H. E., Villringer, A., et al. (2011). Combined evaluation of FDG-PET and MRI improves detection and differentiation of dementia. PLoS One 6:e18111. doi: 10.1371/journal.pone. 0018111

Ehrenberg, A. J., Nguy, A. K., Theofilas, P., Dunlop, S., Suemoto, C. K., Lorenzo Alho, A. T., et al. (2017). Quantifying the accretion of hyperphosphorylated tau in the locus coeruleus and dorsal raphe nucleus: the pathological building blocks of early Alzheimer's disease. Neuropathol. Appl. Neurobiol. 43, 393-408. doi: 10.1111/nan.12387

Feinstein, D., Kalinin, S., and Braun, D. (2016). Causes, consequences, and cures for neuroinflammation mediated via the locus coeruleus: noradrenergic signaling system. J. Neurochem. 139, 154-178. doi: 10.1111/jnc.13447

Fernandes, P., Regala, J., Correia, F., and Gonçalves-Ferreira, A. J. (2012). The human locus coeruleus 3-D stereotactic anatomy. Surg. Radiol. Anat. 34, 879-885. doi: 10.1007/s00276-012-0979-y

German, D. C., Walker, B. S., Manaye, K., Smith, W. K., Woodward, D. J., and North, A. J. (1988). The human locus coeruleus: computer reconstruction of cellular distribution. J. Neurosci. 8, 1776-1788. doi: 10.1523/JNEUROSCI.0805-01776.1988

Giorgi, F., Ryskalin, L., Ruffoli, R., Biagioni, F., Limanaqi, F., Ferrucci, M., et al. (2017). The neuroanatomy of the reticular nucleus locus coeruleus in Alzheimer's Disease. Front. Neuroanat. 11:80. doi: 10.3389/fnana.2017.00080

Grinberg, L., Rüb, U., Ferretti, R., Nitrini, R., Farfel, J., Polichiso, L., et al. (2009). The dorsal raphe nucleus shows phospho-tau neurofibrillary changes before the transentorhinal region in Alzheimer's disease. A precocious onset?. Neuropathol. Appl. Neurobiol. 35, 406-416. doi: 10.1111/j.1365-2990.2009. 00997.x

Grudzien, A., Shaw, P., Weintraub, S., Bigio, E., Mash, D., and Mesulam, M. (2007). Locus coeruleus neurofibrillary degeneration in aging, mild cognitive impairment and early Alzheimer's disease. Neurobiol. Aging 28, 327-335. doi: 10.1016/j.neurobiolaging.2006.02.007

Gulyás, B., Brockschnieder, D., Nag, S., Pavlova, E., Kása, P., Beliczai, Z., et al. (2010). The norepinephrine transporter (NET) radioligand (S,S)[18F]FMeNER-D2 shows significant decreases in NET density in the human brain in Alzheimer's disease: a post-mortem autoradiographic study. Neurochem. Int. 56, 789-798. doi: 10.1016/j.neuint.2010.03.001

Hämmerer, D., Callaghan, M., Hopkins, A., Kosciessa, J., Betts, M., CardenasBlanco, A., et al. (2018). Locus coeruleus integrity in old age is selectively related to memories linked with salient negative events. Proc. Nat. Acad. Sci. U.S.A. 115, 2228-2233. doi: 10.1073/pnas.1712268115

Hannah, J. A. (1936). A case of Alzheimer's disease with neuropathological findings. Can. Med. Assoc. J. 35, 361-366. 
Heneka, M. T., Nadrigny, F., Regen, T., Martinez-Hernandez, A., DumitrescuOzimek, L., Terwel, D., et al. (2010). Locus ceruleus controls Alzheimer's disease pathology by modulating microglial functions through norepinephrine. Proc. Natl. Acad. Sci. U.S.A. 107, 6058-6063. doi: 10.1073/pnas.0909586107

Iglesias, J., Leemput, K., Bhatt, P., Casillas, C., Dutt, S., Schuff, N., et al. (2015). Bayesian segmentation of brainstem structures in MRI. Neuroimage 113, 184-195. doi: 10.1016/j.neuroimage.2015.02.065

Jack, C., Bennett, D., Blennow, K., Carrillo, M., Dunn, B., Haeberlein, S., et al. (2018). NIA-AA research framework: toward a biological definition of Alzheimer's disease. Alzheimers Dement. 14, 535-562. doi: 10.1016/j.jalz.2018. 02.018

Johnston, B., Tolomeo, S., Gradin, V., Christmas, D., Matthews, K., and Steele, D. (2015). Failure of hippocampal deactivation during loss events in treatmentresistant depression. Brain 138, 2766-2776. doi: 10.1093/brain/awv177

Ju, Y.-E., McLeland, J., Toedebusch, C., Xiong, C., Fagan, A., Duntley, S., et al. (2013). Sleep quality and preclinical Alzheimer Disease. JAMA Neurol. 70, 587-593. doi: 10.1001/jamaneurol.2013.2334

Kehoe, E., McNulty, J., Mullins, P., and Bokde, A. (2014). Advances in MRI biomarkers for the diagnosis of Alzheimer's disease. Biomark. Med. 8, 1151-1169. doi: $10.2217 / \mathrm{bmm} .14 .42$

Keren, N., Lozar, C., Harris, K., Morgan, P., and Eckert, M. (2009). In vivo mapping of the human locus coeruleus. Neuroimage 47, 1261-1267. doi: 10.1016/j.neuroimage.2009.06.012

Keren, N., Taheri, S., Vazey, E., Morgan, P., Granholm, A.-C., Aston-Jones, G., et al. (2015). Histologic validation of locus coeruleus MRI contrast in post-mortem tissue. Neuroimage 113, 235-245. doi: 10.1016/j.neuroimage.2015.03.020

Khachaturian, A., Hayden, K., Mielke, M., Tang, Y., Lutz, M., Gustafson, D., et al. (2018). Future prospects and challenges for Alzheimer's disease drug development in the era of the NIA-AA research framework. Alzheimers Dement. 14, 532-534. doi: 10.1016/j.jalz.2018.03.003

Knopman, D., Haeberlein, S., Carrillo, M., Hendrix, J., Kerchner, G., Margolin, R., et al. (2018). The national institute on aging and the Alzheimer's association research framework for Alzheimer's disease: perspectives from the research roundtable. Alzheimers Dement. 14, 563-575. doi: 10.1016/j.jalz.2018.03.002

Kranz, G., Hahn, A., Savli, M., and Lanzenberger, R. (2012). Challenges in the differentiation of midbrain raphe nuclei in neuroimaging research. Proc. Natl. Acad. Sci. U.S.A. 109, E2000-E2000. doi: 10.1073/pnas.1206247109

Kreisl, W. C., Henter, I. D., and Innis, R. B. (2018). Imaging translocator protein as a biomarker of neuroinflammation in dementia. Adv. Pharmacol. 82, 163-185. doi: 10.1016/bs.apha.2017.08.004

Kumar, J. S., and Mann, J. J. (2014). PET tracers for serotonin receptors and their applications. Cent. Nerv. Syst. Agents Med. Chem. 14, 96-112. doi: 10.2174/ 1871524914666141030124316

Lanctôt, K. L., Herrmann, N., and Mazzotta, P. (2001). Role of serotonin in the behavioral and psychological symptoms of dementia. J. Neuropsychiatry Clin. Neurosci. 13, 5-21. doi: 10.1176/jnp.13.1.5

Lanzenberger, R., Mitterhauser, M., Spindelegger, C., Wadsak, W., Klein, N., Mien, L.-K., et al. (2007). Reduced serotonin-1a receptor binding in social anxiety disorder. Biol. Psychiatry 61, 1081-1089. doi: 10.1016/j.biopsych.2006.05.022

Lee, H.-Y., Tae, W., Yoon, H.-K., Lee, B.-T., Paik, J.-W., Son, K.-R., et al. (2011). Demonstration of decreased gray matter concentration in the midbrain encompassing the dorsal raphe nucleus and the limbic subcortical regions in major depressive disorder: an optimized voxel-based morphometry study. J. Affect. Disord. 133, 128-136. doi: 10.1016/j.jad.2011.04.006

Lee, J., Ryan, J., Andreescu, C., Aizenstein, H., and Lim, H. (2015). Brainstem morphological changes in Alzheimer's disease. Neuroreport 26, 411-415. doi: 10.1097/WNR.0000000000000362

Lehto, J., Johansson, J., Vuorilehto, L., Luoto, P., Arponen, E., Scheinin, H., et al. (2015). Sensitivity of [11C]ORM-13070 to increased extracellular noradrenaline in the CNS - a PET study in human subjects. Psychopharmacology 232, 4169-4178. doi: 10.1007/s00213-015-3941-y

Liu, K., Marijatta, F., Hämmerer, D., Acosta-Cabronero, J., Düzel, E., and Howard, R. (2017). Magnetic resonance imaging of the human locus coeruleus: a systematic review. Neurosci. Biobehav. Rev. 83, 325-355. doi: 10.1016/j. neubiorev.2017.10.023

Lyness, S. A., Zarow, C., and Chui, H. C. (2003). Neuron loss in key cholinergic and aminergic nuclei in Alzheimer disease: a meta-analysis. Neurobiol. Aging 24, 1-23. doi: 10.1016/S0197-4580(02)00057-X
Mann, D. M. (1983). The locus coeruleus and its possible role in ageing and degenerative disease of the human central nervous system. Mech. Ageing Dev. 23, 73-94. doi: 10.1016/0047-6374(83)90100-8

Mann, D. M., and Yates, P. O. (1974). Lipoprotein pigments-their relationship to ageing in the human nervous system. II. The melanin content of pigmented nerve cells. Brain 97, 489-498. doi: 10.1093/brain/97.1.489

Marcyniuk, B., Mann, D. M. A., and Yates, P. O. (1986). Loss of nerve cells from locus coeruleus in Alzheimer's disease is topographically arranged. Neurosci. Lett. 64, 247-252. doi: 10.1016/0304-3940(86)90336-8

Matthews, K., Chen, C., Esiri, M., Keene, J., Minger, S., and Francis, P. (2002). Noradrenergic changes, aggressive behavior, and cognition in patients with dementia. Biol. Psychiatry 51, 407-416. doi: 10.1016/S0006-3223(01)01235-5

McKhann, G., Knopman, D., Chertkow, H., Hyman, B., Jack, C., Kawas, C., et al. (2011). The diagnosis of dementia due to Alzheimer's disease: recommendations from the National Institute on Aging-Alzheimer's Association workgroups on diagnostic guidelines for Alzheimer's disease. Alzheimers Dement. 7, 263-269. doi: 10.1016/j.jalz.2011.03.005

Meeten, F., Davey, G., Makovac, E., Watson, D., Garfinkel, S., Critchley, H., et al. (2016). Goal directed worry rules are associated with distinct patterns of amygdala functional connectivity and vagal modulation during perseverative cognition. Front. Hum. Neurosci. 10:553. doi: 10.3389/fnhum.2016.00553

Mehta, D., Jackson, R., Paul, G., Shi, J., and Sabbagh, M. (2017). Why do trials for Alzheimer's disease drugs keep failing? A discontinued drug perspective for 2010-2015. Expert Opin. Inv. Drug 26, 735-739. doi: 10.1080/13543784.2017. 1323868

Miyoshi, F., Ogawa, T., Kitao, S., Kitayama, M., Shinohara, Y., Takasugi, M., et al. (2013). Evaluation of parkinson disease and Alzheimer Disease with the use of neuromelanin MR imaging and 123i-metaiodobenzylguanidine scintigraphy. Am. J. Neuroradiol. 34, 2113-2118. doi: 10.3174/ajnr.A3567

Montine, T., Phelps, C., Beach, T., Bigio, E., Cairns, N., Dickson, D., et al. (2012). National Institute on Aging-Alzheimer's Association guidelines for the neuropathologic assessment of Alzheimer's disease: a practical approach. Acta Neuropath. 123, 1-11. doi: 10.1007/s00401-011-0910-3

Nelson, P., Trojanowski, J., Abner, E., Al-Janabi, O., Jicha, G., Schmitt, F., et al. (2016). "New Old Pathologies": AD, part, and cerebral age-related TDP-43 with sclerosis (CARTS). J. Neuropathol. Exp. Neurol. 75, 482-498. doi: 10.1093/jnen/ nlw033

Nensa, F., Beiderwellen, K., Heusch, P., and Wetter, A. (2014). Clinical applications of PET/MRI: current status and future perspectives. Diagn. Interv. Radiol. 20, 438-447. doi: 10.5152/dir.2014.14008

Neuropathology Group (2001). Pathological correlates of late-onset dementia in a multicentre, community-based population in England and Wales. neuropathology group of the medical research council cognitive function and ageing study (MRC CFAS). Lancet 357, 169-175.

Paterson, L., Kornum, B., Nutt, D., Pike, V., and Knudsen, G. (2013). 5-HT radioligands for human brain imaging with PET and SPECT. Med. Res. Rev. 33, 54-111. doi: 10.1002/med.20245

Phan, J.-A. A., Landau, A. M., Jakobsen, S., Wong, D. F., and Gjedde, A. (2017). Radioligand binding analysis of $\alpha 2$ adrenoceptors with [11C]yohimbine in brain in vivo: extended inhibition plot correction for plasma protein binding. Sci. Rep. 7:15979. doi: 10.1038/s41598-017-16020-1

Pillai, R., Zhang, M., Yang, J., Mann, J., Oquendo, M., Parsey, R., et al. (2018). Molecular connectivity disruptions in males with major depressive disorder. J. Cereb. Blood Flow Metab. doi: 10.1177/0271678X18764053 [Epub ahead of print].

Quarantelli, M. (2015). MRI/MRS in neuroinflammation: methodology and applications. Clin. Transl. Imaging 3, 475-489. doi: 10.1007/s40336-015-0142-y

Quiroz, Y., Sperling, R., Norton, D., Baena, A., Arboleda-Velasquez, J., Cosio, D., et al. (2018). Association between amyloid and tau accumulation in young adults with autosomal dominant Alzheimer Disease. JAMA Neurol. 75, 548-556. doi: 10.1001/jamaneurol.2017.4907

Raja, R., Rosenberg, G., and Caprihan, A. (2017). MRI measurements of blood-brain barrier function in dementia: a review of recent studies. Neuropharmacology 134, 259-271. doi: 10.1016/j.neuropharm.2017. 10.034

Rice, L., and Bisdas, S. (2017). The diagnostic value of FDG and amyloid PET in Alzheimer's disease-A systematic review. Eur. J. Radiol. 94, 16-24. doi: 10.1016/ j.ejrad.2017.07.014 
Robertson, I. (2013). A noradrenergic theory of cognitive reserve: implications for Alzheimer's disease. Neurobiol. Aging 34, 298-308. doi: 10.1016/j. neurobiolaging.2015.09.019

Rüb, U., Tredici, K., Del Schultz, C., Thal, D. R., Braak, E., and Braak, H. (2000). The evolution of Alzheimer's disease-related cytoskeletal pathology in the human raphe nuclei. Neuropathol. Appl. Neurobiol. 26, 553-567. doi: 10.1046/j. 0305-1846.2000.00291.x

Sasaki, M., Shibata, E., Tohyama, K., Kudo, K., Endoh, J., Otsuka, K., et al. (2008). Monoamine neurons in the human brain stem: anatomy, magnetic resonance imaging findings, and clinical implications. Neuroreport 19:1649. doi: 10.1097/ WNR.0b013e328315a637

Sasaki, M., Shibata, E., Tohyama, K., Takahashi, J., Otsuka, K., Tsuchiya, K., et al. (2006). Neuromelanin magnetic resonance imaging of locus ceruleus and substantia nigra in Parkinson's disease. Neuroreport 17:1215. doi: 10.1097/01. wnr.0000227984.84927.a7

Schain, M., Tóth, M., Cselényi, Z., Arakawa, R., Halldin, C., Farde, L., et al. (2013). Improved mapping and quantification of serotonin transporter availability in the human brainstem with the HRRT. Eur. J. Nucl. Med. Mol. Imaging 40, 228-237. doi: 10.1007/s00259-012-2260-3

Sclocco, R., Beissner, F., Bianciardi, M., Polimeni, J., and Napadow, V. (2017). Challenges and opportunities for brainstem neuroimaging with ultrahigh field MRI. Neuroimage 168, 412-426. doi: 10.1016/j.neuroimage.2017.02.052

Silverberg, N., Elliott, C., Ryan, L., Masliah, E., and Hodes, R. (2018). NIA commentary on the NIA-AA research framework: towards a biological definition of Alzheimer's disease. Alzheimers Dement. 14, 576-578. doi: 10.1016/ j.jalz.2018.03.004

Šimić, G., Babić Leko, M., Wray, S., Harrington, C. R., Delalle, I., JovanovMilošević, N., et al. (2017). Monoaminergic neuropathology in Alzheimer's disease. Prog. Neurobiol. 151, 101-138. doi: 10.1016/j.pneurobio.2016.04.001

Singh-Manoux, A., Dugravot, A., Fournier, A., Abell, J., Ebmeier, K., Kivimäki, M., et al. (2017). Trajectories of depressive symptoms before diagnosis of dementia: a 28-year follow-up study. JAMA Psychiatry 74, 712-718. doi: 10.1001/ jamapsychiatry.2017.0660

Sommerauer, M., Fedorova, T. D., Hansen, A. K., Knudsen, K., Otto, M., Jeppesen, J., et al. (2018). Evaluation of the noradrenergic system in Parkinson's disease: an 11C-MeNER PET and neuromelanin MRI study. Brain 141, 496-504. doi: 10.1093/brain/awx348

Son, Y.-D., Cho, Z.-H., Choi, E.-J., Kim, J.-H., Kim, H.-K., Lee, S.-Y., et al. (2014). Individually differentiated serotonergic raphe nuclei measured with brain PET/MR imaging. Radiology 272, 541-548. doi: 10.1148/radiol.14131547

Sperling, R., Aisen, P., Beckett, L., Bennett, D., Craft, S., Fagan, A., et al. (2011). Toward defining the preclinical stages of Alzheimer's disease: recommendations from the National Institute on Aging-Alzheimer's Association workgroups on diagnostic guidelines for Alzheimer's disease. Alzheimers Dement. 7, 280-292. doi: $10.1016 /$ j.jalz.2011.03.003

Stehouwer, J., and Goodman, M. (2009). Fluorine-18 radiolabeled PET tracers for imaging monoamine transporters: dopamine, serotonin, and norepinephrine. Pet Clin. 4, 101-128. doi: 10.1016/j.cpet.2009.05.006

Steuwe, C., Daniels, J., Frewen, P., Densmore, M., Theberge, J., and Lanius, R. (2015). Effect of direct eye contact in women with PTSD related to interpersonal trauma: psychophysiological interaction analysis of connectivity of an innate alarm system. Psychiatry Res. Neuroimaging 232, 162-167. doi: 10.1016/j. pscychresns.2015.02.010

Szabadi, E. (2013). Functional neuroanatomy of the central noradrenergic system. J. Psychopharmacol. 27, 659-693. doi: 10.1177/026988111349 0326

Szot, P., Leverenz, J., Peskind, E., Kiyasu, E., Rohde, K., Miller, M., et al. (2000). Tyrosine hydroxylase and norepinephrine transporter mRNA expression in the locus coeruleus in Alzheimer's disease. Mol. Brain Res. 84, 135-140. doi: 10.1016/S0169-328X(00)00168-6
Takahashi, J., Shibata, T., Sasaki, M., Kudo, M., Yanezawa, H., Obara, S., et al. (2015). Detection of changes in the locus coeruleus in patients with mild cognitive impairment and Alzheimer's disease: high-resolution fast spin-echo T1-weighted imaging. Geriatr. Gerontol. Int. 15, 334-340. doi: 10.1111/ggi. 12280

Tan, C.-C. C., Yu, J.-T. T., and Tan, L. (2014). Biomarkers for preclinical Alzheimer's disease. J. Alzheimers Dis. 42, 1051-1069. doi: 10.3233/JAD-140843

Tejani-Butt, S., Yang, J., and Zaffar, H. (1993). Norepinephrine transporter sites are decreased in the locus coeruleus in Alzheimer's disease. Brain Res. 631, 147-150. doi: 10.1016/0006-8993(93)91201-3

Theofilas, P., Ehrenberg, A., Dunlop, S., Alho, A., Nguy, A., Leite, R., et al. (2017). Locus coeruleus volume and cell population changes during Alzheimer's disease progression: a stereological study in human postmortem brains with potential implication for early-stage biomarker discovery. Alzheimers Dement. 13, 236-246. doi: 10.1016/j.jalz.2016.06.2362

Tona, K.-D., Keuken, M., Rover, M., de Lakke, E., Forstmann, B., Nieuwenhuis, S., et al. (2017). In vivo visualization of the locus coeruleus in humans: quantifying the test-retest reliability. Brain Struct. Funct. 222, 4203-4217. doi: 10.1007/ s00429-017-1464-5

Trillo, L., Das, D., Hsieh, W., Medina, B., Moghadam, S., Lin, B., et al. (2013). Ascending monoaminergic systems alterations in Alzheimer's disease. Translating basic science into clinical care. Neurosci. Biobehav. Rev. 37, 1363-1379. doi: 10.1016/j.neubiorev.2013.05.008

Villemagne, V., Doré, V., Burnham, S., Masters, C., and Rowe, C. (2018). Imaging tau and amyloid- $\beta$ proteinopathies in Alzheimer disease and other conditions. Nat. Rev. Neurol. 14, 225. doi: 10.1038/nrneurol.2018.9

Wilson, R., Nag, S., Boyle, P., Hizel, L., Yu, L., Buchman, A., et al. (2013). Neural reserve, neuronal density in the locus ceruleus, and cognitive decline. Neurology 80, 1202-1208. doi: 10.1212/WNL.0b013e3182897103

Wolk, D., and Detre, J. (2012). Arterial spin labeling MRI: an emerging biomarker for Alzheimer's disease and other neurodegenerative conditions. Curr. Opin. Neurol. 25, 421-428. doi: 10.1097/WCO.0b013e328354ff0a

World Health Organization (2017). Global Action Plan on the Public Health Response to Dementia 2017-2025. Geneva: World Health Organization.

Zarow, C., Lyness, S., Mortimer, J., and Chui, H. (2003). Neuronal loss is greater in the locus coeruleus than nucleus basalis and substantia nigra in Alzheimer and Parkinson diseases. Arch. Neurol. 60, 337-341. doi: 10.1001/archneur.60.3.337

Zecca, L., Casella, L., Albertini, A., Bellei, C., Zucca, F. A., Engelen, M., et al. (2008). Neuromelanin can protect against iron-mediated oxidative damage in system modeling iron overload of brain aging and Parkinson's disease. J. Neurochem. 106, 1866-1875. doi: 10.1111/j.1471-4159.2008.05541.x

Zhang, X., Yang, Z., Lu, G., Yang, G., and Zhang, L. (2017). PET/MR imaging: new frontier in alzheimer's disease and other dementias. Front. Mol. Neurosci. 10:343. doi: 10.3389/fnmol.2017.00343

Zhou, J., Greicius, M., Gennatas, E., Growdon, M., Jang, J., Rabinovici, G., et al. (2010). Divergent network connectivity changes in behavioural variant frontotemporal dementia and Alzheimer's disease. Brain 133, 1352-1367. doi: 10.1093/brain/awq075

Conflict of Interest Statement: The authors declare that the research was conducted in the absence of any commercial or financial relationships that could be construed as a potential conflict of interest.

Copyright (c) 2018 Braun and Van Eldik. This is an open-access article distributed under the terms of the Creative Commons Attribution License (CC BY). The use, distribution or reproduction in other forums is permitted, provided the original author(s) and the copyright owner(s) are credited and that the original publication in this journal is cited, in accordance with accepted academic practice. No use, distribution or reproduction is permitted which does not comply with these terms. 